\title{
A novel method for the automatic evaluation of retinal vessel tortuosity
}

Enrico Grisan, Marco Foracchia, and Alfredo Ruggeri

Department of Information Engineering, University of Padova, Italy

This work was partially supported by a research grant from Nidek Technologies, Italy. 


\begin{abstract}
Tortuosity is among the first alterations in retinal vessel network to appear in many retinopathies. Automatic evaluation of retinal vessel tortuosity is thus a valuable tool for early detection of vascular suffering. Quite a few techniques for tortuosity measurement and classification have been proposed, but they do not always match the clinical concept of tortuosity. This justifies the need for a new definition, able to express in mathematical terms the tortuosity as perceived by ophthalmologists. We propose here a new algorithm for the evaluation of tortuosity in vessels extracted from digital fundus images. It is based on the partitioning of each vessel in segments of constant-sign curvature and on the combination between the number of such segments and their curvature values. This algorithm has been compared with the other tortuosity measures on a set of 20 vessels from 10 different images. These vessels had been preliminarily ordered by an expert ophthalmologist in order of increasing perceived tortuosity. The proposed algorithm proved to be the best one as regards arterial tortuosity and among the best for vein tortuosity evaluation.
\end{abstract}

\title{
Index Terms
}

Tortuosity, vessels, fundus imaging, retina, curvature.

\section{INTRODUCTION}

Many diseases, such as diabetes and hypertension, have the retina as target organ, and some are only recognizable by changes in the vascular network of the retina (retinopathy of prematurity). One of the first changes to occur is the increasing in vessel tortuosity. In order to evaluate clinical significance of tortuosity changes with time, or to compare different levels of retinopathy, there is a strong need to develop a tortuosity measure that matches the clinical perception of ophthalmologists. At first, we have to clearly understand the main factors that influence the classification of a vascular structure as tortuous or non-tortuous. This is even more important in analyzing retinal images, in which not only straight vessels but also long vessels presenting a smooth semi-circular shape are considered as non-tortuous. The need to develop a numerical index incorporating these factors arose from the observation that previously proposed methods (see [1] for a review) failed in differentiating the tortuosity of structures that visually appeared to be very different in tortuosity, as it will be shown in Sec. III.

\section{TORTUOSITY PROPERTIES}

Vessel tortuosity measures do not have a formal clinical definition, but common practice has outlined an evaluation of the tortuosity with some well-defined properties. In order to obtain a clinically meaningful vessel tortuosity measure, i.e. a measure that is able to match the ophthalmologist's evaluation, it is necessary to make these properties explicit. Then, using a proper mathematical formulation of the tortuosity functional, the proposed measure must be shown to satisfy these properties.

\section{A. Affine Transformations}

A sound approach to extract properties of a not formally defined index is to perform a preliminary study on the invariance properties of such index with respect to the most relevant transformation in the domain space. In 
particular, we will consider affine transformations of a vessel: translation, rotations and scaling.

Translation and rotation transformation are not supposed to influence the perception of tortuosity. In fact, these transformation are related to the geographical position and orientation of vessels in the retina, and do not alter in any way the clinical perception of tortuosity. Scaling of the single vessels does not seem to affect the clinical perception of tortuosity, but a warning flag should be raised since different clinicians may have different opinions about this issue. The scaling is particularly controversial when considering also the vessel calibre, but for the purpose of evaluating single vessel tortuosity, its perception can be safely considered invariant to scaling.

\section{B. Composition}

Composition properties deal with the behavior of tortuosity perception when two vessel curves are merged into a single curve, or when various segments of the same vessel, in general with different tortuosity measures, build up to give the total vessel tortuosity.

Given two adjacent continuous curves $s_{1}$ and $s_{2}$, we define the combination of the two as:

$$
s_{3}=s_{1} \oplus s_{2}
$$

Since the two composing curves are supposed to belong to the same vessel, we can assume the continuity of $s_{3}$ without loss of generality.

In [1], an intuitive empirical principle was proposed:

$$
\tau\left(s_{1}\right) \leq \tau\left(s_{2}\right) \Rightarrow \tau\left(s_{1}\right) \leq \tau\left(s_{1} \oplus s_{2}\right) \leq \tau\left(s_{2}\right)
$$

which means that, when composing two curves, the resulting tortuosity is between those of the composing curves. The counterexample shown in Fig. 1, top panel, clearly show that this statement can not be accepted in conjunction with the principle of invariance with respect to rotation and scale: two curves (e.g., $L_{1}$ and $L_{2}$ ) perceived by themselves as almost non tortuous, when connected form an undoubtedly tortuous curve.

Therefore we propose a new composition property, such that a vessel $s$, combination of various segments $s_{i}$, will not have tortuosity measure less than any of its composing parts:

$$
\begin{array}{r}
\tau\left(s_{i}\right) \leq \tau\left(s_{1} \oplus s_{2} \oplus \ldots \oplus s_{n}\right) \\
\forall i=1 \ldots n \mid s_{i} \subseteq s
\end{array}
$$

\section{Modulation}

It is useful now to express a monotonic relationships with respect to two other properties, which we will call frequency modulation at constant amplitude and amplitude modulation at constant frequency. It may be assumed that the greater the number of changes in the curvature sign (twist) is, the more tortuous the vessel can be considered. Similarly, the greater the amplitude (maximum distance of the curve from the underlying chord) of a twist is, the greater is the tortuosity associated with it. 
For two vessels having twists with the same amplitude, the difference in tortuosity varies with the number of twists $\varphi$ :

$$
\varphi\left(s_{1}\right) \leq \varphi\left(s_{2}\right) \Rightarrow \tau\left(s_{1}\right) \leq \tau\left(s_{2}\right)
$$

and, conversely, for two vessels with the same number of twists (with the same frequency), the difference in tortuosity depends on the difference in amplitude $\alpha$ of the twists:

$$
\alpha\left(s_{1}\right) \leq \alpha\left(s_{2}\right) \Rightarrow \tau\left(s_{1}\right) \leq \tau\left(s_{2}\right)
$$

\section{Available tortuosity measures}

Various tortuosity measures have been proposed in the literature, but all fails in certain aspects. In this section we will review the available methods for evaluating retinal vessel tortuosity and will present some counterexamples in which these methods provide results that do not match with clinical perception.

\section{A. Arc Length over Length ratio}

The simpler and most widely used measure of a vessel tortuosity is the ratio between its length and the length of the underlying chord [1] [2] [3]. The idea of using this ratio is that the greater the value of the ratio, the more distant the vessel is from a straight line, i.e., tortuous. Unfortunately, being the surface of the retina close to a semi-sphere, the non tortuous paradigm should be the circle arc. In fact, every vessel that has a constant and small curvature, regardless of the amplitude of the arc it describes (as for the main retinal vessels), will be regarded by an ophthalmologist as of negligible tortuosity. Moreover, it is shown in Fig. 1 that two vessels with very different tortuosity have the same arc length over length ratio measure.

\section{B. Measure involving curvature}

Hart [1] presented a number of tortuosity measures that involve the use of the integral of the absolute curvature or of the squared curvature. For a curve $s(l)=[x(l), y(l)]: D \subset \mathbb{R} \rightarrow \mathbb{R}^{2}, s \epsilon C^{1}(\mathbb{R})$, with $l$ the curvilinear coordinate on it, the curvature $C_{s}(l)$ is:

$$
C_{s}(l)=\frac{\dot{x}(l) \ddot{y}(l)-\ddot{x}(l) \dot{y}(l)}{\left(\dot{x}^{2}(l)+\dot{y}^{2}(l)\right)^{\frac{3}{2}}}
$$

The idea behind this is that this integral should be a measure of the variability of vessel direction. However, the example presented in Fig. 1 shows that a smaller curvature integral may correspond to a greater perceived tortuosity. In our opinion, there are three main reasons for this result. The first is that curvature is non zero only along arcs, while it is negligible along straight or almost straight segments; but straight segments together with arcs dramatically change the tortuosity appearance. The second is that changes in convexity (curvature sign) of the curve are not taken into account. On the contrary, this is the main feature used by expert graders to assess tortuosity. Finally, integrating along domain possibly different in dimension yields to measures depending on the aforesaid dimension. The ratios between the absolute curvature integral (or the squared curvature integral) and chord length (or vessel length) have been proposed to circumvent the latter point, but still the other two problems remain open with this definition of tortuosity. 

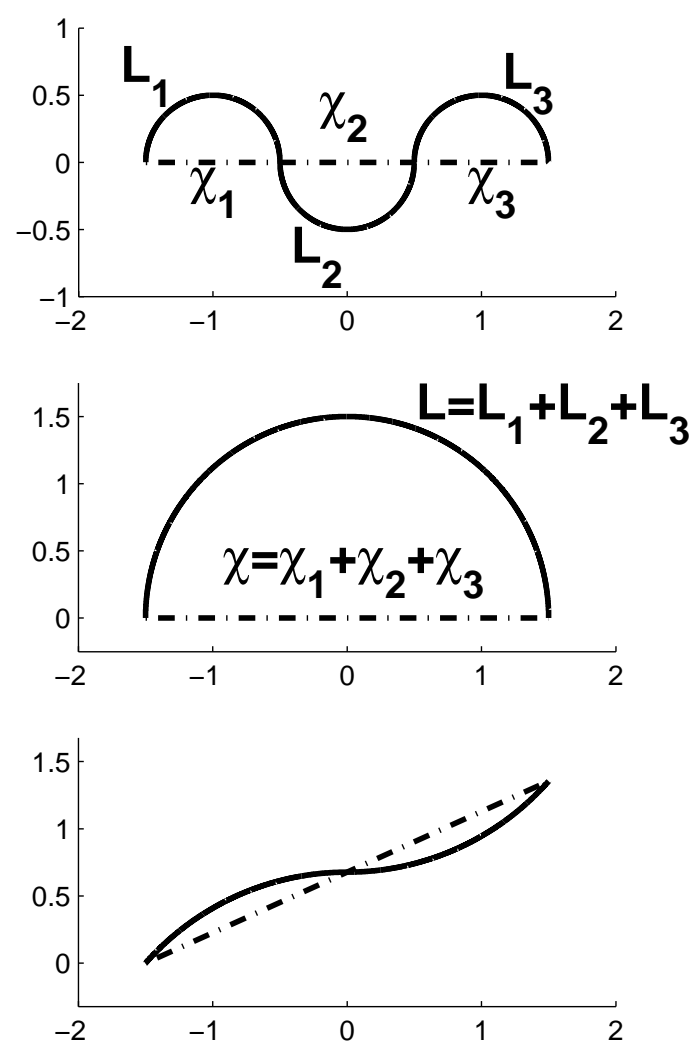

Fig. 1. The first two curves (top and middle panel) have very different tortuosity but the same Length $L$ and Chord Length $\chi$. The second and last curves (middle and bottom panel) have the same average angular difference despite their different tortuosity. The curve in the bottom panel has a curvature integral of $\pi / 2$ whereas the one in the middle panel has curvature integral $\pi$, even if that in the bottom panel is clearly perceived as more tortuous.

\section{Mean direction angle change}

More recently, a measure of tortuosity based on a local directional changes of the vessel has been proposed [4]. It computes the average of the angles between sample points describing the vessel. For each point $i$ of the vessel, it computes the versors from the previous step point $\boldsymbol{d}_{i-s t e p}$ and to the subsequent point $\boldsymbol{d}_{i+s t e p}$ :

$$
a d_{s}=\frac{1}{L_{c}-2 * s t e p} \sum_{i=1}^{n} \arccos \left(\boldsymbol{d}_{\boldsymbol{i}-\boldsymbol{s t e p}} \cdot \boldsymbol{d}_{\boldsymbol{i}+\boldsymbol{s t e p}}\right)
$$

In addition to the high sensitivity to noise, both from the digital quantization and from the vessel extraction technique, it suffers of the problems mentioned above. Even the simple example of Fig. 1 shows that a vessel with constant curvature, such as a semi-circumference, and a vessel formed by the juxtaposition of two arcs of circumference have the same average angle variation, despite their difference in tortuosity. Moreover, also here vessel segments with no variation in direction do not weight in the tortuosity. 


\section{Methodology}

\section{A. Available Data}

The theoretical mathematical description of the vessel is a curve in a two dimension space:

$$
s(l): D \subset \mathbb{R} \rightarrow \mathbb{R}^{2}
$$

The available description of the vessel is a sampled, quantized and noise corrupted version of the theoretical curve:

$$
s(k): s\left(l_{k}\right)
$$

where $l_{k}$ is a sequence of curvilinear coordinates that represent the sampling on the original vessel.

For this study, a set of 20 vessels from 10 different retinal images was used. Images were acquired with a fundus camera with a $50^{\circ}$ field of view and then digitized with a scanner at 1300x1100 pixels. Vessel segments were automatically extracted by a previously developed tracking algorithm [5].

\section{B. Vessel Representation}

Sampling of a vessel may lead to a very sparse vessel description, since the sampling strategy may vary greatly with the vessel profile acquisition from digital images. Sparse description of the vessel further leads to a poor description of its dynamics, which may eventually fail to provide useful information on vessel direction and its derivatives. Sampling can be taken care of by means of an interpolation function, which describes the vessel between sampling points, where no data are present. Physiologically, vessels are structures continuous at least with their first derivative and this condition leads to the use of a cubic spline interpolation. Since the data are noisy, splines also offer the opportunity of filtering by using cubic smoothing splines.

The resulting spline curve is obtained from the available data by regularization:

$$
\hat{s}=\arg \min _{\hat{s}}\left[(1-\gamma) E_{i}(\hat{s})+\gamma\left\|\hat{s}-s_{q}\right\|\right]
$$

where $E_{i}(s)$ is what is called internal energy term, and represents the smoothness of the curve; $\gamma$ is a weighting parameter, varying between 0 and 1 , that sets the compromise between following the available data $s_{q}$ and having a 'smooth' behavior. For $\gamma=1 \hat{s}$ is the least square linear fit of the data, whereas for $\gamma=0$ it is the variational cubic spline interpolant of the data. In this study we used $\gamma=0.005$.

\section{Tortuosity Calculation}

When evaluating tortuosity, ophthalmologists integrate information about how many times a vessel twists (changes in convexity, or curvature sign), and how large is the amplitude of each of the recognized twist. We would therefore decompose any curve into a set of consecutive segments of constant curvature sign.

Having defined the curvature of a curve in (5), we define a subsegment $s_{i}$ of $s$ as a turn curve if:

$$
\left[C_{s_{i}}(l) \geq 0, \forall l \in D\right] \vee\left[C_{s_{i}}(l) \leq 0, \forall l \in D\right]
$$

In real images, it is common to find small oscillations (changes of convexity with very small amplitude) around the main vessel direction, due to the presence of noise. These oscillations might affect the correct decomposition of 
the vessel, since they would create a great number of artificial turn curves, which are not clinically significant. An hysteretic threshold on curvature was thus used to deal with these small variations, as shown in Fig. 2. To evaluate

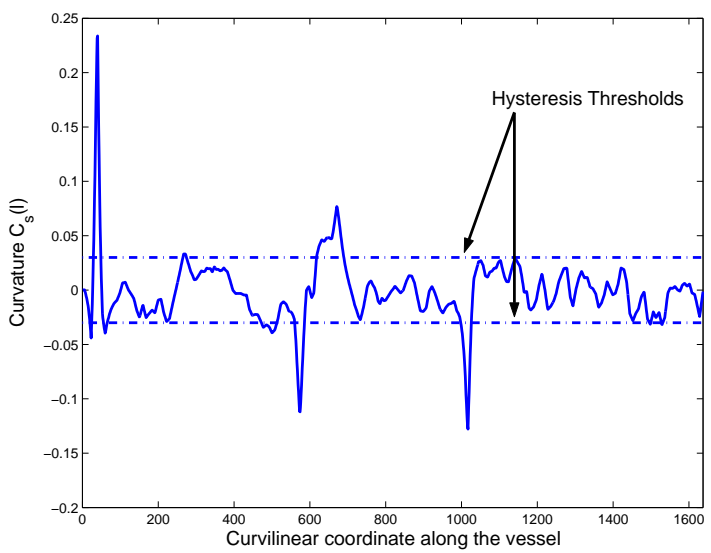

Fig. 2. Curvature value along a vessel. The dashed lines indicate the hysteretic threshold for the convexity change

tortuosity, we also need the Chord Length $L_{\chi}$ of a curve, defined as the distance between the two extremes of the it:

$$
L_{\chi}=\|s(\max (D))-s(\min (D))\|
$$

and the Curve Length $L_{c}$, defined as:

$$
L_{c}=\int_{\min (D)}^{\max (D)}\left\|\frac{\partial s}{\partial l}\right\| d l
$$

An hysteretic threshold of 0.03 has been set for arteries and one of 0.01 for veins. The two types of vessels have been kept separate, since ophthalmologists grade arteries and veins differently. Due to the possible presence of turn curves with zero curvature, the decomposition is not unique. Since eliminating these turn curves might lead to the situation in which an increase in amplitude with straight segments would not increase the tortuosity, we chose to split the straight segments into two halves, assigning one to the preceding and one to the following turn curve.

Once a curve $s(l)$ is divided into $n$ turn curves

$$
s_{i}: s=s_{1} \oplus s_{2} \oplus \ldots \oplus s_{n}
$$

we propose a new definition of vessel tortuosity as:

$$
\tau(s)=\frac{n-1}{L_{c}} \sum_{i=1}^{n}\left[\frac{L_{c_{s_{i}}}}{L_{\chi_{s_{i}}}}-1\right]
$$

This tortuosity measure has a dimension of $1 /$ length and thus may be interpreted as a tortuosity density, allowing its comparison on vessels of different length. It is worth noting that when $n$ is equal to 1 then $\tau$ is equal to 0 and thus vessels with a constant convexity have zero tortuosity.

The proposed definition of tortuosity meets all the properties described in Sec. II. In particular, the composition property is satisfied via the summation in (14), the amplitude modulation via the ratio of the length over the chord 
length for every turn curve, and the frequency modulation is taken care of both implicitly by means of the curve splitting and explicitly through the multiplicative term $n-1$ in (14).

\section{RESUlts AND Discussion}

From the set of 10 images with various degree of vascular tortuosity, 13 arterial and 7 venous segments have been automatically tracked. The selected vessels have then been proposed to an expert ophthalmologist, who ordered them with increasing tortuosity. The proposed method was then compared with those presented in Sec. III, for which, calling $\kappa$ the curvature of a curve, we define as in [1] the total curvature and the total squared curvature as the integral of $|\kappa|$ and $\kappa^{2}$, respectively. Computation of $\kappa$ involves the evaluation of first and second derivatives along the curve, which was done both by numerical differentiation and by the same spline representation of the vessel used for the computation of the proposed measure $\tau$.

$$
\begin{aligned}
t c & =\int_{\min D}^{\max D}|\kappa(l)| d l \\
t s c= & \int_{\min D}^{\max D}\left|\kappa(l)^{2}\right| d l
\end{aligned}
$$

In Table I and Table II values of the correlation with the clinician perception for all the tortuosity indexes mentioned in this paper are shown. These results show that the proposed measure has the best correlation as regards artery tortuosity and is among the three best ones as regards vein tortuosity.

\begin{tabular}{|c|c|c|}
\hline Tortuosity Measure & Arteries & Veins \\
\hline$L_{c} / L_{\chi}$ & 0.857 & 0.036 \\
tc & -0.113 & -0.464 \\
tsc & -0.146 & 0.286 \\
$t c / L_{c}$ & 0.011 & -0.036 \\
$t s c / L_{c}$ & -0.015 & 0.286 \\
$t c / L_{\chi}$ & 0.149 & -0.107 \\
$t s c / L_{\chi}$ & -0.008 & 0.321 \\
$a d$ & -0.323 & 0.714 \\
$\tau$ & 0.890 & 0.857 \\
\hline \multicolumn{3}{|c}{ TABLE I }
\end{tabular}

CORRELATION OF THE TORTUOSITY MEASURES WITH THE OPHTHALMOLOGIST GRADING; CURVATURE FOR $t c$ AND $t s c$ OBTAINED BY NUMERICAL

$$
\text { DIFFERENTIATION }
$$

\section{CONCLUSION}

We have proposed a new algorithm for evaluating tortuosity of retinal vessels. It has proved to be reliable and robust, and it does not suffer from the theoretical drawbacks of the tortuosity measures previously proposed. A more extensive evaluation, based on a larger set of images and vessels, will be performed shortly, in order to obtain results with higher statistical reliability. 


\begin{tabular}{|c|c|c|}
\hline Tortuosity Measure & Arteries & Veins \\
\hline$L_{c} / L_{\chi}$ & 0.857 & 0.036 \\
tc & 0.542 & 0.071 \\
tsc & 0.712 & 0.607 \\
$t c / L_{c}$ & 0.778 & 0.929 \\
$t s c / L_{c}$ & 0.817 & 0.929 \\
$t c / L_{\chi}$ & 0.831 & 0.750 \\
$t s c / L_{\chi}$ & 0.857 & 0.750 \\
$a d$ & -0.323 & 0.714 \\
$\tau$ & 0.890 & 0.857 \\
\hline \multicolumn{3}{|c}{ TABLE II }
\end{tabular}

CORRELATION OF THE TORTUOSITY MEASURES WITH THE OPHTHALMOLOGIST GRADING; CURVATURE FOR $t c$ AND $t s c$ BY SPLINE REPRESENTATION

\section{ACKNOWLEDGMENT}

The authors would like to thank Prof. S. Piermarocchi and colleagues at the Department of Ophthalmology, University of Padova, for providing images and clinical advice.

Marco Foracchia is now with $M^{2}$ Scientific Computing, Italy.

\section{REFERENCES}

[1] W. E. Hart, M. Goldbaum, B. Côté, P. Kube, and M. R. Nelson, "Measurement and classification of retinal vascular tortuosity," International Journal of Medical Informatics, vol. 53, no. 2-3, pp. 239-252, February 1999.

[2] C. Heneghan, J. Flynn, M. O'Keefe, and M. Cahill, "Characterization of changes in blood vessel width and tortuosity in retinopathy of prematurity using image analysis," Medical Image Analysis - In Press - Available online 8 February 2002.

[3] M. E. Martinez-Perez, A. D. Hughes, A. V. Stanton, S. A. Thom, N. Chapman, A. A. Bharath, and K. H. Parker, "Retinal vascular tree morphology: a semi-automatic quantification," IEEE Transactions on Biomedical Engineering, vol. 49, no. 8, pp. 912-917, August 2002.

[4] K. V. Chandrinos, M. Pilu, R. B. Fisher, and P. E. Trahanias, "Image processing techniques for the quantification of atherosclerotic changes," in Proceedings MEDICO98, Limassol, Cyprus, June 1998.

[5] M. Foracchia, E. Grisan, and A. Ruggeri, "Quantitative description of vessel features in hypertensive retinopathy fundus images," in Abstracts of the 2nd CAFIA workshop, 2001, p. 15. 